\title{
Lumen
}

Selected Proceedings from the Canadian Society for Eighteenth-Century Studies

\section{Un mythe libéral, une invention des Lumières : Le commerce comme remède à la guerre}

\section{Stéphane Pujol}

Volume 30, 2011

URI : https://id.erudit.org/iderudit/1007719ar

DOI : https://doi.org/10.7202/1007719ar

Aller au sommaire du numéro

Éditeur(s)

Canadian Society for Eighteenth-Century Studies / Société canadienne d'étude du dix-huitième siècle

ISSN

1209-3696 (imprimé)

1927-8284 (numérique)

Découvrir la revue

Citer cet article

Pujol, S. (2011). Un mythe libéral, une invention des Lumières : Le commerce comme remède à la guerre. Lumen, 30, 113-130.

https://doi.org/10.7202/1007719ar

Copyright (C Canadian Society for Eighteenth-Century Studies / Sociéte canadienne d'étude du dix-huitième siècle, 2011
Ce document est protégé par la loi sur le droit d'auteur. L'utilisation des services d'Érudit (y compris la reproduction) est assujettie à sa politique d'utilisation que vous pouvez consulter en ligne.

https://apropos.erudit.org/fr/usagers/politique-dutilisation/ 


\section{Un mythe libéral, une invention des Lumières : Le commerce comme remède à la guerre}

\section{Introduction}

Dans la perspective qui est celle de la philosophie politique à l'âge classique, les relations entre les peuples sont conçues comme le prolongement des rapports État-société. C'est à cette condition que peuvent être combattus l'état de guerre et l'anarchie sur la scène internationale. Dans le discours du courant libéral tel qu'il s'exprime aujourd'hui, cette pétition de principe semble se décliner à travers trois tendances qui découlent directement des idéaux des Lumières : pour les uns, c'est avant tout la nature du régime politique qui dicte le comportement des États en matière de relation internationale, et par conséquent le recours à la guerre ou à la coopération : la paix serait alors garantie par l'instauration générale de la démocratie «libérale» (les démocraties ne faisant pas la guerre entre elles, selon la notion de «paix démocratique» inspirée de Rousseau et de Kant et reprise plus tard par Wilson) ; pour d'autres, ce sont essentiellement les échanges commerciaux qui favorisent la coopération : transposant la thèse des «besoins mutuels» de Montesquieu et adaptant la théorie de «la main invisible» d'Adam Smith dans le cadre des relations internationales, le libéralisme économique considère qu'il y a possibilité de convergence entre les intérêts des puissances souveraines ; pour les derniers, la stabilité et la paix doivent être garanties par des institutions internationales fondées en droit (c'est le projet de l'abbé de Saint-Pierre et de Kant).

Il semble que parmi les diverses solutions que la philosophie des Lumières a élaborées pour empêcher la guerre entre les nations et pour imaginer sous un jour pacifique les relations internationales, la plus controversée est bien celle du commerce. On s'interroge de nos jours 
pour savoir si la forme moderne de l'internationalisation des échanges, appelée «mondialisation», est de nature à favoriser la paix. Si l'on veut retracer la genèse de cette idée «libérale», en saisir les nuances et la complexité, il faut évidemment convoquer l'auteur de l'Esprit des Lois. Dans un passage fameux de son œuvre maîtresse, Montesquieu défend l'idée que la paix passe par le développement des échanges commerciaux et par la dépendance réciproque des peuples que les premiers entraînent. Le commerce, écrit-il, "guérit des préjugés destructeurs ; [...] partout où il $\mathrm{y}$ a des mœurs douces, il $\mathrm{y}$ a du commerce ; partout où il y a du commerce, il y a des mœurs douces ${ }^{1} . »$ Selon Montesquieu, mais ce sera le credo de nombreux philosophes des Lumières, les échanges économiques, par le biais du «besoin mutuel» des nations qui en découle, seraient un formidable facteur d'apaisement et de pacification des relations internationales.

Je voudrais interroger cette thèse et quelques-uns de ses présupposés à la lumière des événements historiques, et notamment des conflits internationaux qui caractérisent la deuxième moitié du XVIII siècle. Le commerce est-il toujours représenté comme un jeu à somme positive? Le recours à la violence n'est-il pas aussi une source ou une conséquence du commerce ; le commerce n'est-il pas, déjà, une guerre d'intérêts?

On est surpris de constater que la thèse du commerce pacificateur a survécu à la guerre de Sept $\mathrm{Ans}^{2}$. Il suffit de lire l'Histoire philosophique et politique des deux Indes de l'abbé Raynal pour s'en convaincre. Comment l'expliquer? La réponse se trouve, semble-t-il, dans l'analyse de ses enjeux.

Après avoir brièvement considéré les rapports du commerce et de la guerre dans une perspective historique, nous essaierons de montrer dans quelle mesure l'idée du commerce comme remède à la guerre est à la fois un idéal - c'est-à-dire un objet non empiriquement démontrable — et une idéologie : idéal et idéologie que l'épithète «libéral» simplifie plutôt qu'il ne les enrichit.

1 De l'Esprit des lois, Livre XX, chapitre 1.

2 Rappelons que la guerre de Sept Ans (1756-1763) est le premier conflit mondial. Il concerne à la fois les frontières de l'Europe et les possessions françaises et anglaises dans le reste du monde, et notamment en Amérique du Nord. 


\section{Guerre et commerce au XVIII ${ }^{\mathrm{e}}$ siècle.}

\subsection{La guerre au service du commerce.}

Le XVII ${ }^{\mathrm{e}}$ siècle avait été un siècle de guerre. Le XVIII ${ }^{\mathrm{e}}$ siècle se déroulera également au rythme des conflits ${ }^{3}$. Après la guerre de Trente Ans et la paix de Westphalie (1648), l'Europe avait pu croire à un début de répit. Il dura en effet jusqu'à la guerre de succession d'Espagne (17111713) qui ouvre ce que les historiens ont appelé une "seconde guerre de Cent Ans», durant laquelle l'Angleterre et la France luttent pour l'hégémonie continentale et mondiale. Avec le traité d'Utrecht (1713), le difficile partage des possessions coloniales en Amérique du Nord sera source de tensions et de différends grandissants qui déboucheront sur la guerre de Sept Ans (1756-1763). Ce nouveau conflit francobritannique a pour conséquence l'exportation des théâtres de guerre hors du continent européen. Celui-ci se trouve ainsi à l'origine d'une internationalisation des rapports de force entre les différentes nations. Parallèlement, le modèle contractuel du traité étend son influence, et à travers lui une certaine uniformisation du droit international se dessine ${ }^{4}$. Dix traités d'alliance sont signés par l'Angleterre et la France entre la paix d'Utrecht et la déclaration de guerre de $1793^{5}$. Désormais, la guerre relève plus directement de la diplomatie et de la sphère politique. Elle se sécularise tandis que sa dimension éthique devient plus relative. Alors que la guerre était traditionnellement associée à un acte de justice vindicative, en tant que punition d'une première injustice, elle s'apparente de plus en plus à une violation du droit. Sa dimension juridique en sortira paradoxalement renforcée. Comme le rappelle l'historien du droit Jean-Mathieu Mattéi, «le positionnement opposant état de paix et état de guerre est caractéristique du droit naturel. (...)

3 Entre 1700 et 1819, l'Europe connaît un total de 46 années de guerre continentale, sans compter les conflits mineurs. Le nombre de militaires tués pour la France pour cette période est environ de 2,1 million (500 000 pour la guerre de succession d'Espagne, 140000 pour celle de l'Autriche ; 175000 pour la guerre de sept ans ; 500000 pour la Révolution ; 800000 pour l'Empire. Nous tirons nos sources du Dictionnaire d'art et d'histoire militaire, PUF, 1988, p. 670 et sq.

4 On l'appelle au XVIII siècle le «droit public extérieur».

5 Voir Georg Friedrich Martens, Cours diplomatique ou Tableau des relations extérieures des puissances de l'Europe ... , tome III, Paris, Levreault, 1801, Livre I, chap. IV, §54, p. 63-64. 
L'état de paix devient ainsi synonyme de sociabilité, de concorde, d'union et de communications raisonnables entre États ${ }^{6} . »$

En matière de théorie des relations internationales, on considère qu'il n'existe en pratique que trois systèmes : l'hégémonie impériale, l'équilibre des puissances et la sécurité collective. Au XVIII ${ }^{\mathrm{e}}$ siècle, la première appartient déjà au passé, et la troisième, à travers les différents projets de paix perpétuelle, est à peine en cours d'élaboration ${ }^{7}$. Il faut par ailleurs rappeler que les relations internationales sont encore régies selon l'ordre westphalien, «ordre amoral où les intérêts nationaux priment sur les considérations éthiques» et «où la souveraineté des États est absolue ${ }^{8} »$. Au XVIII ${ }^{\mathrm{e}}$ siècle, les traités de paix ont également un rapport étroit avec la question du commerce (ils sont d'ailleurs souvent rédigés et présentés conjointement), mais ils sont différemment appréhendés selon les politiques nationales. S'ils permettent aux États de consolider leur puissance, ils relèvent parfois de stratégies contraires. Comme le signale l'économiste J.-F. Melon, le jeu de la concurrence va pousser les Anglais et les Français dans deux directions inverses : «l'esprit de la nation anglaise est de tourner les traités mêmes en commerce, et l'esprit de la nation française est de tourner le commerce même en traités ${ }^{9}$.»

Le jeu de la concurrence est encore avivé par les politiques mercantilistes qui règnent des deux côtés de la Manche. Dans la perspective qui est celle du mercantilisme, l'ouverture au commerce international ne vise pas au rapprochement des nations mais participe plutôt d'une

6 Jean-Mathieu Mattéi, Histoire du droit de la guerre (1700-1819): introduction à l'histoire du droit international, PU Aix-Marseille, 2006, p. 89, note 83. Cette partie de notre réflexion s'inspire de cette importante enquête historique.

7 C'est à la promotion de celui-ci que s'attacheront des penseurs comme l'abbé de Saint-Pierre, Rousseau et Kant ; mais avec la guerre de Sept Ans, le système de la «balance des puissances» qu'il faudrait préserver retrouve tout son crédit. Un Mémoire de 1770 intitulé Moyens à employer par la France pour parvenir à replacer l'Angleterre au point qu'elle doit occuper dans la balance de l'Europe, souligne le fait que l'Angleterre ne doit sa puissance «ni à son sol, ni à sa population, ni à la seule industrie, mais à son commerce presque exclusif ... ». Notons toutefois que dans l'esprit de ce publiciste, parce qu'elle ne repose que sur le commerce, cette puissance est «factice» et elle ne permet pas d'inférer une possible suprématie politique.

8 Arnaud Blin, 1648, La paix de Westphalie ou la naissance de l'Europe politique moderne, Editions Complexe, Paris, 2006, p. 18.

9 J.-F. Melon, Essai politique sur le commerce, in Collection des principaux économistes, édition procurée par Eugène Daire, Paris, Guillaumin, 1843, p. 730. 
logique d'affrontement. Pour les tenants de ce système, non seulement la guerre se met au service du commerce, mais le commerce lui-même est conçu comme une guerre où «l'un ne peut ordinairement gagner que l'autre ne perde ${ }^{10} . »$

\subsection{Le commerce au service de la guerre.}

Au cours des guerres internationales menées par l'Angleterre de 1689 à 1815 , la solidité de ses finances fut un élément capital de son accession au rang de puissance mondiale. Le commerce anglais a prospéré pendant la guerre de Sept $\mathrm{Ans}^{11}$. Son commerce colonial, tout comme celui de la France, est devenu considérable. Il a stimulé les industries nationales qui exportaient, mais il a également encouragé la production de bois et de charpentes destinés à construire à la fois les navires de commerce et les vaisseaux de guerre. Cet aspect de la puissance anglaise est mis en évidence par Voltaire dans la dixième de ses Lettres philosophiques (1734) : «c'est le commerce qui a établi peu à peu les forces navales par qui les Anglais sont les maîtres des mers.» On connaît l'éloge que fait Voltaire du commerce anglais. On connaît moins «l'anti-lettre philosophique» que constituent les deux premiers chapitres de ses Fragments sur l'Inde. Dans ce texte tardif (1773), loin de souscrire à la thèse du commerce pacificateur, le philosophe évoque en termes cinglants la guerre de Sept Ans, résultat selon lui de «l'avidité des marchands, encore plus que de l'ambition des souverains ${ }^{12}$.» Et il ajoute un peu plus loin :

Le commerce, ce premier lien entre les hommes, étant devenu un objet de guerre, et un principe de dévastation, les premiers mandataires des Compagnies [des Indes] Anglaise et Française, salariés par leurs commettants sous le nom de Gouverneurs, furent bientôt des espèces de Généraux d'armée : on les aurait pris dans l'Inde pour des Princes ; ils faisaient la guerre et la paix tantôt entre eux, tantôt avec les souverains de ces contrées ${ }^{13}$.

10 Mercure de France, avril 1720, cité par Catherine Larrère dans L'invention de l'économie au XVIIIe siècle : du droit naturel à la physiocratie, Paris, Presses Universitaires de France, 1992, p. 102.

11 François Crouzet, La guerre économique franco-anglaise au XVIII siècle, Paris, Fayard, 2008, p. 360.

12 Voltaire, Fragments sur l'Inde, sur le général Lally et sur le comte de Morangiés, Article premier, dans Tableau historique du Commerce de l'Inde, édition de 1773, p. 4-5.

13 Ibid, Article second, p. 10. 
L'exemple anglais est donc ambivalent : le développement de la puissance navale britannique a permis l'extension des conquêtes coloniales. Inversement, les progrès de la guerre ont stimulé le commerce et l'industrie du pays. Il y a là un schéma logique que les philosophes vont vouloir questionner : le commerce favorise la guerre qui favorise le commerce ... On peut bien prendre cette chaîne causale à un autre moment (la guerre favorise le commerce qui favorise la guerre, etc.), les effets sont les mêmes. Autrement dit, on fait la guerre pour des raisons commerciales ; on fait du commerce pour des motifs militaires. Il me semble précisément que les Lumières vont renverser cette chaîne de causalité - selon une logique moins historique que théorique ou anthropologique - en plaçant comme premier vecteur non pas la guerre, mais la paix (la paix favorise le commerce qui favorise la paix ... ). Ce schéma à la fois linéaire et circulaire a pu longtemps faire illusion. On comprend qu'il s'agit d'un modèle idéal et abstrait. On voit en effet rarement les philosophes examiner dans le détail le jeu des causalités et se demander si le commerce est réellement cause ou effet de la paix.

Cette question prend un tour dialectique dans la tragédie de Charles IX (1789) de Marie-Joseph Chénier ${ }^{14}$. Transposant le conflit qui oppose la France aux puissances de l'Europe monarchique dans le contexte des guerres de religion du $\mathrm{XVI}^{\mathrm{e}}$ siècle, la pièce de Chénier souligne le fait que la soif de conquête et les rivalités commerciales sont toujours prétextes à de nouvelles guerres. Elle semble faire un sort à cette contradiction d'un commerce «paisible» qui enfante néanmoins la guerre :

Le paisible Commerce enfantera la guerre

Mais ramenant les rois à leurs vrais intérêts,

Le besoin du Commerce enfantera la paix.

Et cent peuples, rivaux de gloire et d'industrie,

Unis et rapprochés, n'auront qu'une patrie ${ }^{15}$.

Les rivalités commerciales engendrent la guerre, mais la nécessité du commerce entraîne inéluctablement le retour de la paix. La fonction du commerce paraît dès lors équivoque, à la fois source de conflits et motif de résolution des conflits. Par delà la coquetterie poétique du vers de Chénier (on aura noté l'oxymore : le paisible commerce qui enfante la guerre), le schéma logique a gagné en complexité : il va du commerce

14 Charles IX ou la Saint Barthélémy, [1789] tragédie en cinq actes (rebaptisée Charles IX ou l'Ecole des Rois en 1790).

15 Marie-Joseph Chénier, Charles IX, ou la Saint-Barthélémy, Acte II, scène 3. 
à la guerre, de la guerre à la paix, de la paix au commerce. Les deux chaînes de causalité paraissent finalement se croiser. C'est peut-être la variante littéraire d'un paradigme philosophique que l'on retrouvera plus tard avec Kant, celui du remède dans le mal.

\section{La thèse du commerce pacificateur.}

\section{1 «'Lesprit de commerce» chez Montesquieu}

Les temps de paix sont bien des temps d'échange, sinon de prospérité et de développement économique. A contrario, comme le souligne Montesquieu,

Le commerce, tantôt détruit par les conquérants, tantôt gêné par les monarques, parcourt la terre, fuit d'où il est opprimé, se repose où on le laisse respirer : il règne aujourd'hui où l'on ne voyait que des déserts, des mers et des rochers ; là où il régnait, il n'y a que des déserts ${ }^{16}$.

Mais dire que les temps de paix sont propices au commerce revient-t-il à dire que le commerce est pacifique?

On peut partir de cette pétition de principe que l'on trouve dans le chapitre 2 du livre XX de l'Esprit des lois, intitulé «Des lois dans le rapport qu'elles ont avec le commerce considéré dans sa nature et ses distinctions» :

L'effet naturel du commerce est de porter à la paix. Deux nations qui négocient ensemble se rendent réciproquement dépendantes : si l'une a intérêt d'acheter, l'autre à intérêt de vendre; et toutes les unions sont fondées sur les besoins mutuels ${ }^{17}$.

Il faut rappeler, après Albert Hirschman, le rôle de l'intérêt dans la construction du discours sur le commerce au XVIII ${ }^{\mathrm{e}}$ siècle $^{18}$. Le com-

16 Montesquieu, De l'Esprit des Lois, livre XXI, chap. V.

17 Montesquieu, De l'Esprit des Lois, livre XX, chap. II.

18 Nous reviendrons plus loin sur l'ouvrage d'A. O. Hirschmann, The Passions and the Interests : political arguments for capitalism before its triumph, Princeton University Press, Princeton, 1977, traduction française par Pierre Andler, Paris, Presses Universitaires de France, 2001. Il faut aussi signaler les travaux fondateurs de Francine Markovitz, Catherine Larrère, et plus récemment de Céline Spector. 
merce a longtemps été méprisé dans la culture occidentale. Le negotium n'était-il pas la négation de l'otium, ce temps à soi qu'était le loisir antique? L'homme semblait devoir se réaliser hors de la simple recherche de l'intérêt. Dans ses Lois, Platon ne confiait le commerce qu'aux seuls "pérégrins»" ${ }^{19}$. Pour que ce préjugé change, il a fallu penser que le commerce possédait une valeur autre que purement économique. Les grandes découvertes, le développement du commerce entre les continents qui marquent l'ère moderne ont été les conditions historiques de cette réhabilitation. Le commerce a-t-il été pour autant le ferment de nouvelles relations internationales? C'est la thèse répandue au $\mathrm{XVIII}^{\mathrm{e}}$ siècle sous l'expression de «doux commerce» dont le postulat, formulé en d'autres termes par Montesquieu, est partagé par bon nombre de philosophes des Lumières ${ }^{20}$. Dans cette perspective, l'activité lucrative, conduite rationnellement, met un frein à l'expression débridée des passions : «le commerce polit et adoucit les mours barbares» affirme Montesquieu. De façon pragmatique, les guerres ou les conflits ont un coût économique qui appelle la tempérance.

Depuis le Traité de l'économie politique (1715) d'Antoine de Montchrétien, on est habitué à voir dans la paix un instrument du commerce, et non l'inverse. L'originalité de Montesquieu est d'avoir renversé la perspective. Pour l'auteur de l'Esprit des Lois, le commerce est une pratique, mais c'est avant tout un «esprit», c'est-à-dire une disposition intellectuelle qui dépasse le simple cadre de l'échange marchand ou matériel. Le souci accordé aux intérêts de chacune des parties confère au commerce une dimension humaniste puisqu'il s'agit de considérer l'existence de l'autre et de compter avec lui, dans tous les sens du terme. Là où il y a commerce, il y a relation entre deux parties qui souhaitent s'entendre et se mettre d'accord. C'est déjà le commencement de la paix. Encore faut-il que le commerce soit porté par «des besoins mutuels». Albert Hirschman a souligné la manière dont la philosophie des Lumières a développé le thème de l'harmonie des intérêts. Sous des formes distinctes, on trouve en effet chez de nombreux auteurs l'idée que la défense de l'intérêt des particuliers participe à l'intérêt général. Mais cette harmonie n'est pas le fruit d'une intention ou d'une volonté a priori. C'est le principe de convergence involontaire des intérêts que

19 Platon, Lois, VIII, 849d.

20 Une lecture hâtive de Hirschman pourrait faire penser que l'expression de «doux commerce» se trouve sous la plume de Montesquieu. Bien que la «douceur» du commerce soit un paradigme central chez cet auteur, on ne trouve nulle part ce syntagme dans son œuvre. 
l'on va retrouver chez Mandeville et chez Adam Smith (avec la fameuse image de la main invisible développée par Smith dans La richesse des nations), et qui est déjà fortement présent dans la philosophie politique de Montesquieu. Il ne faut pas donc pas chercher d'intention altruiste dans la démarche du commerçant ${ }^{21}$. L'intérêt des parties est un principe moteur : «si l'une a intérêt à acheter, l'autre a intérêt de vendre ${ }^{22}$.»

La guerre de Sept ans commence véritablement au moment où Montesquieu publie l'Esprit des lois. Aurait-il changé de point de vue s'il avait rédigé son œuvre maîtresse quelques années plus tard? Rien n'est moins sûr (lors de sa dernière révision du texte en 1758, il ne modifie rien sur le sujet $\left.{ }^{23}\right)$. En dépit de ce qui est souvent affirmé, son analyse nous semble reposer moins sur des faits contingents que sur la combinaison d'un principe anthropologique et d'une lecture historique qui s'efforce de penser la modernité.

Il importe ici de distinguer "commerce» et «esprit de commerce». Ce qui change en effet avec l'Esprit des lois, c'est la promotion de l'esprit de commerce, notion que l'on retrouvera plus tard chez Adam Smith, Benjamin Constant et même Kant. Cette représentation est le produit d'une idéologie largement partagée par les philosophes des Lumières mais à laquelle Montesquieu donne un sens nouveau en l'élargissant : parce qu'il s'intéresse aux effets du commerce sur une vaste échelle - celle des relations internationales - Montesquieu lui confère une dimension véritablement politique et même cosmopolitique.

21 Sur ce principe de convergence involontaire des intérêts, nous renvoyons au beau travail de Céline Spector, Montesquieu et l'émergence de l'économie politique, Paris, Honoré Champion, 2006.

22 Montesquieu, Esprit des Lois, XX, 2.

23 Cela étant, on peut se demander si l'antériorité de la publication du texte par rapport à la guerre de Sept ans ne force pas un peu le texte. Ainsi, lorsque Montesquieu trouve dans le modèle anglais l'illustration d'un mouvement historique qui voit en même temps l'abandon de l'esprit de conquête et l'essor de l'ordre marchand : «Si cette nation habitait une île, elle ne serait point conquérante, parce que des conquêtes séparées l'affaibliraient. Si le terrain de cette île était bon, elle le serait encore moins, parce qu'elle n'aurait pas besoin de la guerre pour s'enrichir. $\mathrm{Et}$, comme aucun citoyen ne dépendrait d'un autre citoyen, chacun ferait plus de cas de sa liberté que de la gloire de quelques citoyens, ou d'un seul» (Esprit des Lois, XIX, 27). On a en effet bien du mal à voir dans l'Angleterre qui combat la France sur le continent et dans les colonies un exemple de nation à la fois pacifique et commerçante. 
Au moment où il rédige l'Esprit des Lois, l'illusion produite par la paix d'Utrecht ne suffit pas. Renversant la perspective communément admise, Montesquieu ne comprend plus les rapports de la guerre et du commerce comme complémentaires mais comme antagonistes.

Il faut par ailleurs relever l'orientation polémique du livre $X X$ de l'Esprit des Lois. La séparation implicite entre esprit de conquête et esprit de commerce est à replacer dans la perspective de son refus des théories mercantilistes ${ }^{24}$. Montesquieu veut montrer que le commerce, ou plutôt l'esprit de commerce, unit les nations par le même motif qu'il unit les particuliers. Parler de «l'esprit» du commerce, par analogie avec l'esprit d'un individu, c'est d'abord faire signe vers ce qui en constitue l'unité. C'est ensuite indiquer le principe qui sous-tend l'action des hommes. Pour les individus, l'esprit de commerce ne conduit pas simplement à la paix, il produit ce que Montesquieu appelle un sentiment de «justice exacte» :

L'esprit de commerce produit dans les hommes un certain sentiment de justice exacte, opposé d'un côté au brigandage, et de l'autre à ces vertus morales qui font qu'on ne discute pas toujours ses intérêts avec rigidité, et qu'on peut les négliger pour ceux des autres ${ }^{25}$.

24 On notera que cette opposition, que Montesquieu a pu trouver lui-même chez J.-F. Melon (1675-1738) pour qui «l'esprit de Conquête et de Commerce s'excluent mutuellement dans une Nation» (Essai politique sur le commerce, [s. 1.], 1734, chap. 7, p. 97), se manifeste diversement chez les philosophes du courant «libéral». Alors qu'elle est purement chronologique chez Constant, elle semble à la fois logique et chronologique chez Montesquieu. Pour Constant, l'esprit de conquête et l'esprit de commerce correspondent à deux âges successifs de l'humanité. Si Montesquieu semble tendre dans ce sens ses Pensées, (après l'esprit de gloire et de valeur, «c'est l'esprit de commerce qui domine aujourd'hui», Pensées, 810), dans l'Esprit des Lois, la notion «d'esprit» permet aux deux logiques de coexister dans le temps, au gré de la diversité des mœurs politiques et des coutumes. Cette opposition se retrouve encore déplacée chez un lecteur italien de Montesquieu : Antonio Genovesi oppose fortement guerre et commerce, mais insiste au contraire sur la parenté qui unit l'esprit de commerce et l'esprit de conquête tout en précisant que l'esprit du Commerce est l'esprit de conquérir des richesses, pas des pays, ni des personnes : «(..) l'esprit du Commerce n'est rien d'autre que l'esprit de Conquête. Les barbares conquièrent les personnes et les terres : les peuples commerçants conquièrent les richesses», Antonio Genovesi, Delle lezioni di commercio, o sia d'economia civile. Con Elementi del Commercio, Naples, 1766-1767, rééd. 1768-1770), chap. XVII. Enfin, chez Kant "c'est l'esprit de commerce qui ne peut coexister avec la guerre qui tôt ou tard s'empare de chaque peuple» (Vers la paix perpétuelle, VIII, 368, trad. Jean-François Poirier et Françoise Proust, éd. G-F, 1991, p. 107). 
L'adjectif «exacte» peut parait étrange puisqu'une justice inexacte semble être une contradiction dans les termes. Montesquieu tient en fait à préciser que sentiment de justice, dans le cas du commerce, ne relève pas $d^{\prime} u n e$ exigence morale et particulièrement $d$ 'une inclination altruiste qui nous entraînerait à faire plus de cas des autres que de soi. Les «vertus morales» nous amènent, d'une part, à ne pas discuter de notre intérêt de façon stricte (ce qui est le cas de la justice exacte induite par l'esprit de commerce, lequel implique de mesurer de façon quantitative les intérêts de chacun) ; d'autre part à négliger nos intérêts au profit de celui des autres. On peut donc dire que la justice exacte que produit l'esprit de commerce est dénuée de toute moralité. Montesquieu maintient ainsi l'opposition qui deviendra centrale chez Rousseau entre vertu et commerce. À la différence de certains penseurs «libéraux», Montesquieu ne croit pas que le calcul des intérêts peut suppléer à la moralité, mais qu'il permet au contraire de se passer de la vertu.

Par ailleurs, l'harmonie des intérêts postulée au niveau du commerce international $n^{\prime}$ implique nullement $\mathrm{qu}^{\prime}$ on la retrouve au sein d'une même nation. C'est ce qui sépare Montesquieu des économistes ultérieurs comme Gournay ou Smith. Pour Montesquieu, la douceur du commerce est simplement l'effet nécessaire d'une société policée. Mais c'est dans la mesure où la sociabilité est elle-même envisagée comme un «commerce», selon un topos classique, que le lien complexe entre les transactions marchandes et les vertus civilisatrices peut être compris.

\subsection{L'idéal cosmopolite}

Ce que Montesquieu semble inaugurer, c'est un modèle idéal de rapport politique entre les nations dont le commerce est l'un des principaux instruments. On peut dire de ce modèle qu'il est «anti-westphalien», dans la mesure où il permet de dépasser la conception classique de l'État nation : «du point de vue du commerce, le monde entier n'est qu'une seule nation ou un seul peuple, à l'intérieur duquel les nations sont comme des personnes» déclare ainsi Sir Dudley North, dans ses Discourses upon trade rédigés en 1691 . On reconnaît là un des aspects du cosmopolitisme des Lumières, dont la version moderne est celle de la mondialisation des échanges dans le sens d'une «déterritorialisation de l'économie» selon l'expression de Pierre Rosanvallon ${ }^{26}$.

26 Pierre, Rosanvallon, Le libéralisme économique. Histoire de l'idée de marché, Editions du Seuil, 1989, p. 43. 
Ce cosmopolitisme s'affirme tout au long du siècle mais il trouve une illustration particulière dans l'Histoire philosophique et politique des deux Indes de l'abbé Raynal ${ }^{27}$. L'ouvrage, qui se présente à la fois comme une somme encyclopédique et comme une histoire critique et de la colonisation européenne, donne lieu à une véritable apologie du commerce : «en parcourant la terre, en franchissant les mers, en levant les obstacles qui s'opposaient à la communication des peuples, en étendant la sphère des besoins et le désir des jouissances, il multiplie les travaux, il encourage l'industrie, il devient en quelque sorte le moteur du monde ${ }^{28}$. Le commerce apparaît à la fois comme métaphore et comme métonymie d'un processus de civilisation dont l'un des principaux enjeux est la pacification des échanges. Parce qu'il réunit ce que l'espace et le temps séparent, le commerçant devient une figure possible du philosophe. Son action mérite alors d'être saisie à travers un nouveau régime d'écriture de l'histoire : «les annales des peuples demanderont à être écrites par des commerçants philosophes, comme elles l'étaient autrefois par des historiens orateurs» ${ }^{29}$.

Dans l'Esprit des Lois, Montesquieu avait parfaitement mis en évidence la dimension fondamentale des communications dans le développement de l'humanité. L'Histoire des deux Indes de Raynal lui assigne désormais un moment fondateur :

Il n'y a point d'événement aussi intéressant pour l'espèce humaine en général et pour les peuples d'Europe en particulier que la découverte du Nouveau monde et le passage aux Indes par le cap de Bonne-Espérance. Alors a commencé une révolution dans le commerce, dans la puissance des nations, dans les mœurs, $l^{\prime}$ industrie et le gouvernement de tous les peuples. C'est à ce moment que les hommes des contrées les plus éloignées se sont rapprochés par de nouveaux rapports et de nouveaux besoins. Les productions des climats placés sous l'équateur, se consomment dans les climats voisins du pôle ; l'industrie du nord s'est déplacée au sud (...) ; et partout les hommes ont fait un échange mutuel de leurs opinions, de leurs lois, de leurs usages, de leurs maladies, de leurs remèdes, de leurs vertus et de leur vice ${ }^{30}$.

27 Cet ouvrage, dont le titre complet est Histoire philosophique et politique de l'établissement et du commerce des Européens dans les deux Indes, constitue l'un des best sellers de la littérature des Lumières. Il connaît trois éditions successives, en 1770, 1774 et 1780 .

28 Histoire (...) des deux Indes, édition de 1780, Genève, Livre X, p. 231.

29 Ibid, III, p. 328. Ce passage est de la plume de Diderot.

30 Ibid, Livre I, p. 1-2. 
Le commerce entre ainsi dans un mouvement général d'échange et de circulation des hommes, des biens et des idées. Parce qu'il permet au premier chef le rapprochement des peuples, il devient la composante essentielle d'une anthropologie des échanges en cours de constitution au siècle des Lumières. Il n'est certes pas difficile de voir dans la promotion des échanges marchands la justification philosophique du libéralisme économique. Il nous semble également important de rappeler le fait que, dans la perspective des philosophes, le commerce entre dans un rapport étroit avec le cosmopolitisme et par conséquent avec le pacifisme.

Dans un opuscule de 1795, Kant reprend en l'infléchissant la thèse chère à Montesquieu du commerce comme remède à la guerre. Son Projet de paix perpétuelle commence par souligner la nécessité d'une solution «juridique» au problème de la guerre. L'argument du commerce n'intervient que dans le premier Supplément, porté par l'idée selon laquelle le sentiment de «l'intérêt réciproque» conduit les peuples à nouer entre eux des liens que la seule idée du droit cosmopolitique n'aurait pas permis :

De même que la nature sépare sagement les peuples, que la volonté de chaque État, et ce même d'après les fondements du droit des gens, aimerait réunir sous sa domination par la ruse ou la violence, de même elle réunit également, d'autre part, les peuples que le concept de droit cosmopolitique n'aurait pas garantis contre la violence et la guerre, par le biais de leur égoïsme mutuel ${ }^{31}$.

L'action de la nature est ici conçue comme l'effet d'une providence qui, après avoir poussé les hommes à accepter l'idée d'un droit international pour mettre fin aux horreurs de la guerre, les amène à s'unir. Cette perspective téléologique ne peut se comprendre qu'à la lumière de la théorie de «l'insociable sociabilité» développée dans un texte antérieur. Kant l'explique comme «le penchant à entrer en société, lié toutefois à une opposition générale qui menace sans cesse de dissoudre cette société $^{32}$.» Le déchaînement des passions est ainsi le moyen dont

31 Vers la paix perpétuelle (Ak, 368), p. 107.

32 «Le moyen dont se sert la nature pour mener à terme le développement de toutes ses dispositions est leur antagonisme dans la société, dans la mesure où cet antagonisme finira pourtant par être la cause d'un ordre réglé par la loi. J'entends ici par antagonisme l'insociable sociabilité des hommes, c'est-à-dire leur penchant à entrer en société, lié toutefois à une opposition générale qui menace sans cesse de dissoudre cette société». Idée d'une histoire universelle au point de vue cosmopolitique, $4^{\text {ème }}$ proposition, trad. J.-M. Muglioni, Bordas, p. 13-15. 
se sert la nature pour conduire l'humanité vers son propre accomplissement moral. La paix ne procède donc ni de la volonté morale des hommes ni d'une décision politique mais de la guerre elle-même. C'est en quelque sorte un remède dans le mal. Cela ne signifie pas pour autant que Kant considère les relations marchandes comme étant prédisposées par nature à protéger les hommes des conflits. En réalité, son pessimisme anthropologique, qui se représente la nature humaine comme sujette aux passions violentes, lui interdit de souscrire à la thèse du «doux commerce». Il n'est plus question ici d'un adoucissement des mœurs mais simplement d'un calcul rationnel qui place à nouveau la notion d'intérêt réciproque au centre du comportement des hommes. Kant passe ainsi d'une perspective éthique à une perspective pragmatique. Le raisonnement, qui vaut pour les individus, s'applique également aux États. Ceux-ci ne peuvent porter atteinte au commerce sans nuire du même coup à leur propre puissance :

Comme en effet, parmi toutes les puissances (moyens) subordonnées à la puissance de l'État, la puissance d'argent pourrait bien être la plus fiable, les États se voient forcés (certainement pas sous l'effet des mobiles de la moralité) de promouvoir la noble paix, et où que la guerre menace d'éclater dans le monde, de protéger la paix par des interventions, exactement comme si les États se trouvaient engagés, pour cette raison, dans des alliances durables ${ }^{33}$.

Le commerce participe donc d'une forme de régulation extrajuridique dont la raison pratique ne peut cependant pas tout à fait se satisfaire. Comme le remarque justement Dimitri Lang «le schéma téléologique [que Kant] met en avant fait dépendre le pouvoir pacificateur du commerce de l'instauration préalable d'un ordre du droit dans la sphère des relations internationales. C'est avouer que le commerce, loin d'évacuer le droit, en le rendant inutile, ou en le dénonçant comme inefficace, s'appuie en fait sur lui pour répandre ses effets salutaires ${ }^{34}$.» La philosophie de l'histoire de Kant se réalise ainsi dans la tension dialectique entre le penchant des hommes pour la guerre, et leur amour de la liberté qui les pousse à trouver des solutions raisonnables par la voie

34 Dimitri Lang, «Kant et Montesquieu. À propos des vertus pacificatrices du commerce et des relations entre les nations», in Robert Theis et Lukas K. Sosoe (éd.), Les sources de la philosophie kantienne aux XVIIe et XVIIIe siècles, Paris, Vrin, 2005, p. 253-262. 
du commerce ou celle de la législation. C'est alors, selon les propres mots de Kant, que «l'esprit de commerce, qui est incompatible avec la guerre $[. .$.$] s'empare tôt ou tard de chaque peuple».$

Après Montesquieu et après Kant, Benjamin Constant va souligner à son tour la valeur de l'esprit de commerce contre la logique guerrière. Constant va jusqu'à inscrire cette opposition dans une histoire de l'humanité :

Un homme qui serait toujours le plus fort n'aurait jamais l'idée du commerce. [...] La guerre est l'impulsion, le commerce est le calcul. Mais par la-même il doit venir une époque où le commerce remplace la guerre. Nous sommes arrivés à cette époque ${ }^{35}$.

L' «impulsion» s'oppose au calcul, comme l'instinct mal maîtrisé à l'intérêt bien compris. Ce qui sépare le commerce de la guerre, c'est tout l'espace de la culture et la civilisation. Mais pour Benjamin Constant, le commerce entre également dans une histoire générale de la liberté. Il est un moyen pour les individus d'exercer leurs droits sans l'intervention de l'autorité politique ${ }^{36}$. Le texte de Constant insiste sur le désir d'autonomie qui prévaut dans les démocraties modernes, et la volonté des hommes de s'abstraire désormais de la volonté du prince. Le commerce, comme le dit Pierre Manent, est «tout ce qui donne consistance au lien social indépendamment du commandement ${ }^{37} \gg$.

De Montesquieu à Constant, le modèle libéral semble donc participer d'un double mouvement : celui (non explicitement formulé) d'une autonomisation de l'économique ${ }^{38}$; celui d'une régulation normative, c'est-à-dire productrice de valeurs morales, mais sans le secours de la morale. Le commerce, ce serait alors la politique (de la paix) poursuivie par d'autres moyens, pourrait-on dire en détournant la formule

35 De la liberté des Anciens comparée à celle des Modernes, discours prononcé en 1819 à l'Athénée royal de Paris, reproduit par Pierre Manent dans son anthologie intitulée Les Libéraux, éd. Gallimard, coll. «Tel», 2001, p. 443.

36 C'est, pour Constant, le privilège que donne la forme du gouvernement représentatif sur la démocratie directe des Athéniens, qui impliquait la participation constante et active des citoyens au pouvoir politique.

37 Pierre Manent, op. cit., p. 15.

38 C'est la «grande transformation» décrite par Karl Polanyi dans l'ouvrage du même nom : La Grande Transformation. Aux origines politiques et économiques de notre temps [éd. française], Gallimard, 2009. 
de Clausewitz ${ }^{39}$. La sphère économique devient ainsi le lieu où se confondent, pour la première fois, l'ordre des moyens et l'ordre des fins, l'être et le devoir être. À la volonté abstraite de moraliser la vie sociale et politique, se substitue l'argument de la raison pratique - que l'on peut résumer à travers le paradigme de l'intérêt bien compris ; à la logique des rapports de violence et de domination, se substitue celle de l'échange réciproque. La séduction de ce modèle, comme le remarque encore Pierre Rosanvallon, tient à l'aspiration des sociétés modernes «à trouver le moyen de dédramatiser le face-à-face des individus, à dépassionner leurs relations, à désamorcer la violence virtuelle des rapports de force ${ }^{40} »$.

Albert Hirschman a bien montré que cette logique est celle des «justifications politiques du capitalisme avant son apogée». Elle est heureusement résumée par cette formule de Montesquieu : «Il est heureux pour les hommes d'être dans la situation où tandis que leurs passions leur imposent d'être méchants, ils ont cependant intérêt à ne pas l'être ${ }^{41} . »$ Mais si les vertus pacificatrices mises en exergue par les philosophies de l'intérêt ont pu se réaliser, d'autres forces ont joué en sens inverse. Pour paraphraser Marx et Engels, on peut dire que la violence n'a pas été noyée «dans les eaux glacées du calcul égoïste ${ }^{42}$ ».

\section{Conclusion}

Le débat se poursuit aujourd'hui autour de la signification de la mondialisation et son rapport au principe cosmopolitique : faut-il y voir un phénomène $\mathrm{d}$ 'ordre strictement économique et social sans rapport aucun avec l'idéal éthico-juridique du cosmopolitisme des Lumières, ou bien une extension du principe de l'entendement calculateur? N'est-ce pas l'idéal des philosophes cosmopolites, autant que la destinée du concept de commerce, qui méritent ainsi d'être interrogés? Les Lumières sont-elles simplement ordonnées à un projet d'émancipation, ou bien ne sont-elles pas travaillées par une tension théorique interne, lourde de conséquence sur le plan historique?

39 «La guerre n'est que la continuation de la politique par d'autres moyens ...», Clausewitz, De la guerre, chap. I, § 24, éd. Gérard Lebovici, Paris, 1989, p. 51.

40 Pierre Rosanvallon, op.cit., p. V.

41 Montesquieu, De l'Esprit des lois, Livre XXI, chapitre XX.

42 Friedrich Engels et Karl Marx, Manifeste du parti communiste, Paris, Editions sociales, 1966, p. 33. 
Il se peut qu'il y ait, dans l'ensemble complexe qu'on appelle les Lumières, deux formes distinctes de cosmopolitisme : un cosmopolitisme passif et un cosmopolitisme actif. Le premier se repose sur les forces du marché ; il croit à l'intérêt bien compris des individus qui permet de sceller les unions et de développer les échanges ; le second requiert l'adhésion d'une volonté collective qui doit être sanctionnée par le droit. Dans les deux cas, force est de constater chez la plupart des auteurs une relative occultation de la violence coloniale, un "gommage» des rapports de force qui accompagnent certaines formes d'échange marchand. On a beau vouloir lire l'Histoire philosophique et politique des deux Indes de Raynal comme une défense raisonnée, et même raisonnable, de la colonisation, la politique d'expansion commerciale ne va pas sans poser problème ${ }^{43}$.

Faut-il conclure pour autant à la mauvaise foi des Lumières? Ce qui se joue à travers la thèse de la «dépendance réciproque» (Montesquieu) ou de «l'harmonie naturelle des intérêts» (Adam Smith), c'est d'abord une arithmétique de la richesse, de la jouissance et de la prospérité. C'est aussi, nous l'avons vu, la réalisation des libertés individuelles contre la tutelle de la puissance publique. La finalité de cette réalisation est à la fois la régulation de la société civile et la paix entre les nations. Le commerce apparaît alors comme une alternative à la force brute.

Plutôt que de parler de "mauvaise foi», mieux vaut comprendre cette occultation comme le travail de l'idéologie. Le commerce international tel que l'entendent la plupart des philosophes (Rousseau excepté), n'est pas autre chose que l'application d'une conception humaniste $\mathrm{du}$ rapport entre les peuples à la réalité économique ${ }^{44}$. «L'histoire du commerce est celle de la communication des peuples», nous rappelle Montesquieu $^{45}$. Il est aussi une utopie, entendue ici non pas comme un genre de discours littéraire ou philosophique, mais comme une modalité de pensée qui entend prescrire ce qui doit être ${ }^{46}$.

43 Kant s'est également penché sur la question du commerce colonial pour constater sa violence. Mais pour lui la politique coloniale ne contredit pas nécessairement la thèse du commerce pacificateur. Voir Vers la paix perpétuelle (Ak VIII, 359).

44 On peut rappeler la thèse de J.G.A. Pocock, qui voit naître au XVIIIe siècle un «humanisme marchand» relevant «le défi lancé par l'humanisme civique». Voir Vertu, Commerce et Histoire. Essais sur la pensée et l'histoire politique au XVIIIe siècle, Paris, Puf, 1998, p. 242. Montesquieu, De l'Esprit des Lois, XXI, V.

46 Ce lien entre le projet utopique et la prescription de ce qui doit être est clairement 
En réalité, pour un grand nombre de philosophes, la dimension normative et le point de vue pragmatique semblent se confondre ${ }^{47}$. Le commerce tel qu'ils l'analysent pourrait bien désigner par-delà l'échange de biens matériels, une forme de lien social qu'il importe toujours de réactiver. Il n'est pas indifférent en effet que le mot commerce ait pu désigner, à l'âge classique, à la fois la fréquentation des hommes, la circulation des idées et les échanges marchands. Mais ce lien social est fragile, il peut à tout moment se rompre ou se distendre au gré des passions. Et le commerce, comme toute forme d'échange, est aussi le lieu des conflits et des rivalités meurtrières, quand bien même il ne serait pas de son intérêt propre de dégénérer ainsi. La guerre commerciale est une réalité. Le désir d'accumulation des richesses peut ne pas avoir de frein et vouloir renverser, au besoin par la force, tous les obstacles qu'il est susceptible de rencontrer - ce que la tradition marxiste n'a pas manqué de souligner ${ }^{48}$.

Pour les hommes du XVIII ${ }^{e}$ siècle, le commerce est encore, contre la rationalité militaire où les gains des uns impliquent nécessairement les pertes des autres, un jeu à somme positive où tout le monde sort gagnant ${ }^{49}$. S'il apparaît évident que la construction du lien social n'est pas sa finalité première, il produit malgré tout du lien. Dans cette perspective, il faut admettre que les échanges marchands sont non seulement un facteur d'accumulation des richesses, mais aussi un facteur de civilisation. Objet complexe et ambigu, le commerce, avec Montesquieu et avec Kant, s'insère pleinement dans une histoire de l'esprit humain.

STÉPHANE PUJOL

Université de Paris $X$ - Nanterre

Collège international de philosophie

mis en évidence par Kant, (voir Doctrine du Droit in Métaphysique des mœurs, II, Conclusion, trad. A. Renaut, GF-Flammarion, p. 182).

47 C'est encore la posture de Kant (voir Doctrine du Droit, p. 179).

48 C'est ce que rappelle Habermas, qui souligne lui aussi les limites de la thèse kantienne du commerce pacificateur. Voir J. Habermas, La paix perpétuelle. Le bicentenaire d'une idée kantienne, trad. fr. R. Rochlitz, Paris, Le Cerf, 1996, p. 32 et sq.

49 Rationalité militaire que Voltaire élargit en principe anthropologique dans l'article «Patrie» de son Dictionnaire Philosophique : «Telle est la condition humaine, que souhaiter la grandeur de son pays, c'est souhaiter du mal à ses voisins [...]. Il est clair qu'un pays ne peut gagner sans qu'un autre perde». 